INPLASY

PROTOCOL

To cite: Hou et al. Effect of shenmai injection on CancerRelated Fatigue: A protocol for systematic review. Inplasy protocol 202180073. doi: 10.37766/inplasy2021.8.0073

Received: 18 August 2021

Published: 19 August 2021

Corresponding author:

Xianbing Hou

houxianbing007@126.com

Author Affiliation:

Fenghua District Hospital of Traditional Chinese Medicine

Support: None.

Review Stage at time of this submission: Preliminary searches.

Conflicts of interest:

None declared.

\section{Effect of shenmai injection on Cancer-Related Fatigue: A protocol for systematic review}

Hou, X1; Chen, D2; Cheng, X3; Wang, D4; Dai, X5; Wang, Y6; Cui, $\mathrm{B}^{7}$; Wang, $\mathrm{Y}^{8}$; $\mathrm{Xu}, \mathrm{H}^{9}$; Chen, $\mathrm{H}^{10}$.

Review question / Objective: To clarify the efficacy and safety of Shenmai injection in the treatment of cancer - related fatigue.

Condition being studied: The treatment of CRF by traditional Chinese medicine injection (TCMJ) is controversial. We conducted a meta-analysis and systematic review to evaluate the effect of shenmai injection on CRF, with a view to providing some guidance for clinical application.

Information sources: We will start electronic searches from PubMed, MEDLINE, Embase, Web of Science, Cochrane Central Register of Controlled Trials (Central), China National Knowledge Infrastructure (CNKI), China Biomedical Literature Database (CBM), China Science Journal Database (VIP) and Wanfang Database. The search date is from the database to July 30, 2021, and the search language is limited to Chinese and English. In addition, we will manually retrieve other resources, including reference lists of identified publications, conference articles and grey literature.

INPLASY registration number: This protocol was registered with the International Platform of Registered Systematic Review and Meta-Analysis Protocols (INPLASY) on 19 August 2021 and was last updated on 19 August 2021 (registration number INPLASY202180073).

\section{INTRODUCTION}

Review question / Objective: To clarify the efficacy and safety of Shenmai injection in the treatment of cancer - related fatigue
Condition being studied: The treatment of CRF by traditional Chinese medicine injection (TCMJ) is controversial. We conducted a meta-analysis and systematic review to evaluate the effect of shenmai 
injection on CRF, with a view to providing some guidance for clinical application.

\section{METHODS}

Participant or population: Patients diagnosed with cancer-related fatigue will be included regardless of gender, age, race, education and source.

Intervention: Shenmai injection.

Comparator: Conventional treatment.

Study designs to be included: RCTs.

Eligibility criteria: There are clear and recognized diagnostic criteria and efficacy criteria, and all patients are diagnosed as CRF, regardless of gender, age, and origin of the case.

Information sources: We will start electronic searches from PubMed, MEDLINE, Embase, Web of Science, Cochrane Central Register of Controlled Trials (Central), China National Knowledge Infrastructure (CNKI), China Biomedical Literature Database (CBM), China Science Journal Database (VIP) and Wanfang Database. The search date is from the database to July 30, 2021, and the search language is limited to Chinese and English. In addition, we will manually retrieve other resources, including reference lists of identified publications, conference articles and grey literature.

Main outcome(s): Clinical efficacy, including total effective rate or cure rate and fatigue scale score will be accepted as the primary outcomes.

Additional outcome(s): The KPS score, TCM syndrome score, adverse events, will be used as secondary outcomes.

Data management: Data extraction and analysis will be performed independently by two researchers. When differences and opinions are inconsistent, they should be resolved through discussion. If the differences encountered cannot be resolved through discussion, a third author will be invited to resolve them.

Quality assessment / Risk of bias analysis: We will use the "deviation risk" tool in Cochrane Manual v.5.1.0 to assess the deviation risk of each article in the literature. Assessments include equence generation, allocation sequence hiding, blindness of participants and people, and outcome evaluators, incomplete outcome data, selective outcome reporting and other sources of bias. If the risk of bias is high in the literature, we will try to explain and discuss the causes of bias.

Strategy of data synthesis: For dichotomous data, we will denote the outcomes as relative risks (RRs) with $95 \%$ Cls. If the $I^{2}$ test is less than $50 \%$, the fixed effects model will be used for data synthesis. If the $\mathrm{I}^{2}$ test is between $\mathbf{5 0 \%}$ and $75 \%$, the random-effects model will be conducted for data synthesis. If the $I^{2}$ test is higher than $75 \%$, we will investigate possible reasons from both clinical and methodological perspectives, and provide a descriptive analysis or conduct subgroup analysis. For continuous data, if no heterogeneity is detected, we will use mean difference (MD) or standard MD (SMD) to measure the therapeutic effect of $95 \%$ Cls. If significant heterogeneity is found, we will use the random-effects model instead.

Subgroup analysis: If there are obvious heterogeneity, we will conduct subgroup analysis to identify the sources of heterogeneity. We will conduct subgroup analysis according $t \mathrm{o}$ different course time or different outcome indicators.

Sensitivity analysis: When sufficient trials are available, sensitivity analysis will be performed by sequentially eliding each trial to check the robustness of the final results.

Language: None.

Country(ies) involved: China.

Keywords: Cancer-Related Fatigue, shenmai, Injection, systematic review. 
Contributions of each author:

Author 1 - Xianbing Hou.

Email: houxianbing007@126.com

Author 2 - Dandan Chen.

Email: 819328570@qq.com

Author 3 - Tongfei Cheng.

Email: 20964891@qq.com

Author 4 - Dan Wang.

Email: 2537493@qq.com

Author 5 - Xiaojun Dai.

Email: 2398431959@qq.com

Author 6 - Yao Wang.

Email: wangy198302@sohu.com

Author 7 - Bixian Cui.

Email: 459397003@qq.com

Author 8 - Yuanyuan Wang.

Email: wagnyy9208@163.com

Author 9 - Hui Xu.

Email: 598702325@qq.com

Author 10 - Hongzhou Chen.

Email: 2352326376@qq.com 